In Proceedings of Hawaii International Conference on System Sciences (HICSS 2009).

(c) IEEE 2009. Personal use of this material is permitted. However, permission to reprint/republish this material for advertising or promotional purposes or for creating new collective works for resale or redistribution to servers or lists, or to reuse any copyrighted component of this work in other works, must be obtained from the IEEE.

\title{
The Visual Wiki: A New Metaphor for Knowledge Access and Management
}

\author{
Christian Hirsch, John Hosking, John Grundy, Tim Chaffe, David MacDonald, Yuriy Halytskyy \\ University of Auckland, Private Bag 92019, Auckland, New Zealand \\ \{chir008@ec.|john@cs.|john-g@cs.|t.chaffe@|dmac127@ec.|yhal003@ec.\}auckland.ac.nz
}

\begin{abstract}
Successful knowledge management results in a competitive advantage in today's information- and knowledge-rich industries. The elaboration and integration of emerging web-based tools and services has proven suitable for collecting and organizing intellectual property. Due to an increasing information overload, information and knowledge visualizations have become effective methods for representing complex bodies of knowledge in an alternative fashion by using visual languages. The focus of this research is the development of a "Visual Wiki", which combines the notion of a textual and a visual representation of knowledge. A Visual Wiki model has been proposed which provides a unified framework to design and discuss different approaches. Three prototypes of Visual Wikis have been implemented and evaluated according to the improvements to certain knowledge management applications that they facilitate.
\end{abstract}

\section{Introduction}

The "web 2.0", a set of technologies, tools, and concepts, has had an enormous impact on how information is processed using the internet. A more recent trend can be observed as the web 2.0 starts to become incorporated into the enterprise, often referred to as the "enterprise 2.0". Information and knowledge processing in these contexts is becoming more dynamic and with more social aspects than previously. In this domain "wikis" have become very popular knowledge management systems utilizing primarily textual information representations.

Information and knowledge visualizations provide effective methods to represent and convey information- and knowledge-rich scenarios. They support this by providing information in forms more suitable to the human cognitive processing system. In both areas human factors play an important role, including ability to navigate, relate, remember and understand complex information, and possibly to collaboratively share and utilize knowledge. Thus applications in these areas have a significant potential to improve tasks related to knowledge management by making it more accessible, understandable, sharable and dynamic.

This research focuses on the design and implementation of a "Visual Wiki". We define a Visual Wiki to be a combination or integration of two representations of the same underlying body of knowledge - visual and textual - which are sharable and group-editable in a wiki style. Our goal is to improve the capability of a wiki as a knowledge management tool.

The remainder of this paper is organized as follows. Section 2 gives an overview of related research areas. Section 3 and 4 describe the chosen approach and methodology of our research. Central to both is the proposed Visual Wiki model. Section 5 describes the design and implementation of three Visual Wiki prototypes. Section 6 provides an evaluation of those prototypes. Finally section 7 outlines possible future work and section 8 summarizes our conclusions.

\section{Background}

\subsection{Web 2.0 and Enterprise 2.0}

The term "web 2.0" was created by Tim O'Reilly in 2004 [19]. It taxonomises new technologies, application design patterns and business models which have effected enormous changes on how people publish and consume information using the internet. Some of the most important recent applications of the web 2.0 are weblogs, podcasts, RSS feeds, and social software such as wikis, social networking, and social bookmarking. These support rich, dynamic information sharing mechanisms for users. Furthermore combinations of existing web applications and services, called "mashups", are an important part of the new web. Some of the oftenmentioned key characteristics of the web 2.0 are user participation and mass collaboration [22], scalability which leverages the long tail [1], rich user experience, and remixability [19]. 


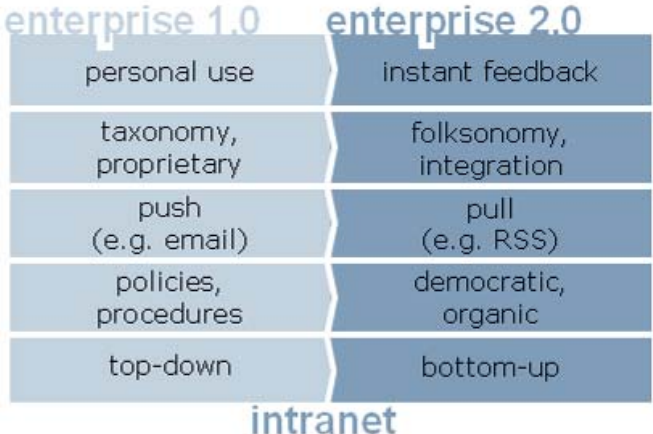

intranet

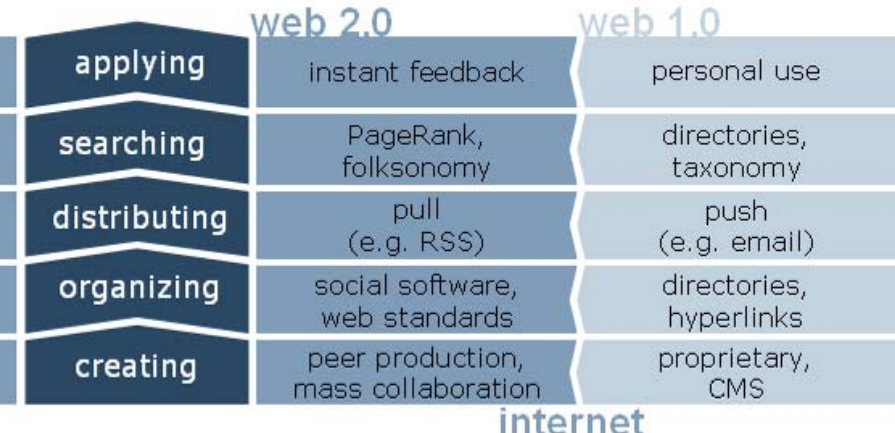

internet

Figure 1. The information lifecycle in the internet and intranet

Enterprises have begun to explore and apply technologies and concepts of the web 2.0 to business intranets. These applications are often referred to as "enterprise 2.0", a term coined by Andrew McAfee [16]. The popularity and importance of these enterprise 2.0 applications are constantly growing [17]. They offer strong potential for managing and developing business information and knowledge assets by providing a new, more democratic, and more evaluative way to exploit information within organizations [24]. Therefore they can have effects on project management, innovation processes, communication patterns, as well as knowledge management [21].

The core concept of the web 2.0 and enterprise 2.0 can be summarized as a gradual and ongoing change in how information is processed. These changes range from the ways information is created and organized via its distribution and search to its application. These five stages of the information lifecycle, as illustrated in Figure 1, can briefly be described as follows.

Fundamental transformation can be observed in the creation of information. The old web (often called "web 1.0") was dominated by proprietary produced information provided by big media or news companies. The web is now gradually becoming a platform of mass collaboration and peer produced content mainly driven by tools like wikis and blogs. In the corporate context this leads to a shift from a top-down to a bottom-up creation of content.

Hyperlinks and web directories based on fixed taxonomies were some of the few means for organizing the information in the web 1.0. In the web 2.0 information is organized in more democratic ways by end users. The resulting "folksonomy" can also be observed in the company environment where it replaces predetermined taxonomies.

In both the internet and the intranet the changes in distribution are mainly about push- versus pulldistribution. More personal and efficient pull distributions such as RSS feeds replace push distributions such as email allowing users to subscribe to what they want and be sent it proactively rather than receiving unsolicited information.

Searching and finding information depends on how well it was organized previously. Search in the old web was dominated by navigating web directories and predefined taxonomies. Search algorithms and folksonomies both created directly or indirectly by the user, are examples of search mechanisms for web 2.0. In enterprise 2.0, tool integration additionally helps to make information more accessible and searchable.

Lastly the application of information is changing from a rather anonymous, personal one-way publication and use to a more social use that allows instant feedback and two-way communication about information. The usage of the information itself can add new information.

The next notable step in this gradual and ongoing change in information processing is arguably called the "web 3.0". It is often referred to as the "semantic web" $[4,8]$ which is seen by many as complementary to the web $2.0[2,10]$.

\subsection{Information \& Knowledge Visualization}

Due to the increasing information overload brought about by the large-scale digitization of information, visualizations have become effective methods for representing and organizing knowledgeand information-rich scenarios [14]. Visualizations themselves are tools for knowledge management which make use of the human cognitive processing system in order to create and convey content more efficiently. Information and knowledge visualization both employ similar techniques. "Based on mapping rules, resource objects are translated into visual objects as meaningful representations, offering easy and comprehensive access to the subject matter presented" [12].

Several models for both domains have been introduced which attempt to describe and classify 
different aspects of the visualization process, most importantly the resource objects, the visual objects and the purpose.

The resource objects in information visualizations are mostly different types of concrete data [13, 20], while those in knowledge visualizations are generally described as more abstract types of knowledge such as declarative knowledge, procedural knowledge, and more [6].

The visual objects are commonly represented using domain specific visual languages (DSVL) such as UML, BPMN, mind maps, and many more [15]. A DSVL uses visualizations to communicate concepts and ideas. Visual objects are here defined as the basic components of any DSVL (e.g. nodes and edges of a graph based DSVL). There is no clear separation between whether a DSVL is used for information or knowledge visualizations. However, information visualizations tend to use rather simple but clearly defined visual languages. Knowledge visualizations use more abstract and flexible ones [6].

Lastly the purpose of visualizations is to support different tasks. Information visualizations focus on concrete tasks such as navigating and filtering an information space [20]. The main task of knowledge visualization is to make knowledge explicit and usable [23]. Further tasks include motivation and elaboration [11]. Knowledge mapping, which is an important and useful type of knowledge visualization, is a good method to capture and share explicit knowledge [26]. This leads to a more general purpose for both information and knowledge visualizations: to support all five steps in the lifecycle (see Figure 1) including the creation, organization, distribution, search, and application of information and knowledge respectively.

\section{Our Approach}

Our approach to this research was to combine the highly dynamic and social concept of the new web with the concept of information visualization. Both concepts are suitable for tasks related to knowledge management. Web 2.0 tools and technologies, such as wikis, are of interest in knowledge management (as summarized in [7]) because of their dynamics and their ability to capture knowledge. Traditional wikis are an editable repository of information, usually predominantly textual, that multiple users share, edit, annotate and use. Human knowledge often exists in flows and can be described as a real time assembly of multiple fragmented memories [7]. A system that supports this kind of loose and ad hoc creation of content is more likely to capture knowledge in an adequate way. Visualizations are tools of knowledge management. They are an alternative and very efficient way to represent and organize knowledgeand information-rich scenarios [6]. Our goal is to combine these two knowledge representations in a "Visual Wiki". In respect to our research we are mainly concerned with explicit knowledge. For the sake of simplicity we will refer to both, "information" and "knowledge" as content which can easily be codified into our prototypes.

A Visual Wiki can be defined as a combination or integration of two representations (a textual and a visual one) of the same underlying body of knowledge. Both or either of the two representations may be editable in a shared, traditional wiki style. The purpose of a Visual Wiki is to increase the capability of a wiki as a knowledge management tool, by elaborating the synergetic effects of integrating visual enhancements.

In the context of the web 2.0, the enterprise 2.0 and their applications, information visualizations are already used in a variety of contexts. Generally they can be found in two formats: active and passive.

Passive visualizations use existing aspects or characteristics of a system, such as tags or page connections in a wiki, visualizing them. Examples are simple tag clouds, wiki structures (e.g. [25]), mashups using wikis and Google Maps (e.g. [3]), or social graphs (e.g. [18]). The resulting visualizations are passive in the way that they are created passively. However, they can be used in an active way, e.g. to navigate a certain structure.

Active visualizations on the other side are realized as rich internet applications allowing the user to create diagrams using a DSVL, and then integrate them with a wiki or other collaboration mechanisms. Examples are the Gliffy diagramming tool (http://gliffy.com) integrated into the Confluence wiki (www.atlassian.com) and Many Eyes [27], a site where users can collaboratively create and discuss visualizations.

The major problem of existing approaches to realizing the concept of a Visual Wiki seems to be a lack of integration between the visual and the textual representations. In other words the approaches are either too active or too passive. One of the major goals of this research is a set of possibilities for overcoming this drawback. In order to not only discuss drawbacks but also to better understand the concept of the Visual Wiki in general, a model is needed. Such a model, as seen in Figure 2, can be used to classify, analyze, and discuss different existing as well as new approaches.

As shown in Figure 2 we define a Visual Wiki to consist of four components: the underlying concept, the textual and visual representation, and a mapping 
between those two. Each of the four components has a set of parameters which can take specific values. In the following, the four components and their most important parameters are described in more detail.

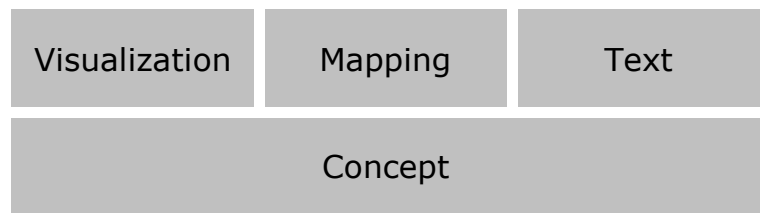

Figure 2. The four components of the Visual Wiki

The concept component, which underlies all of the others, consists of two parameters: purpose and content of the Visual Wiki. (More precisely: purpose and content of the visual component in respect to the whole Visual Wiki). The purpose of the visualization can be any of the five steps in the lifecycle as discussed earlier: creation, organization, distribution, search, and application of knowledge (or any combination of these). The content of the visualization (in respect to the whole body of knowledge contained in the wiki) can range from general to specific.

The text and visualization component are very similar. Both use a language to represent the content of the underlying knowledge base: a visual and a natural language. However, the wiki itself (the textual component) can also be seen as a visual language (e.g. consisting of headlines, text body, tags, etc.). Therefore both components have the same parameters such as complexity, creation, and editing of the metamodel, as well as the creation and editing of the graph and text respectively.
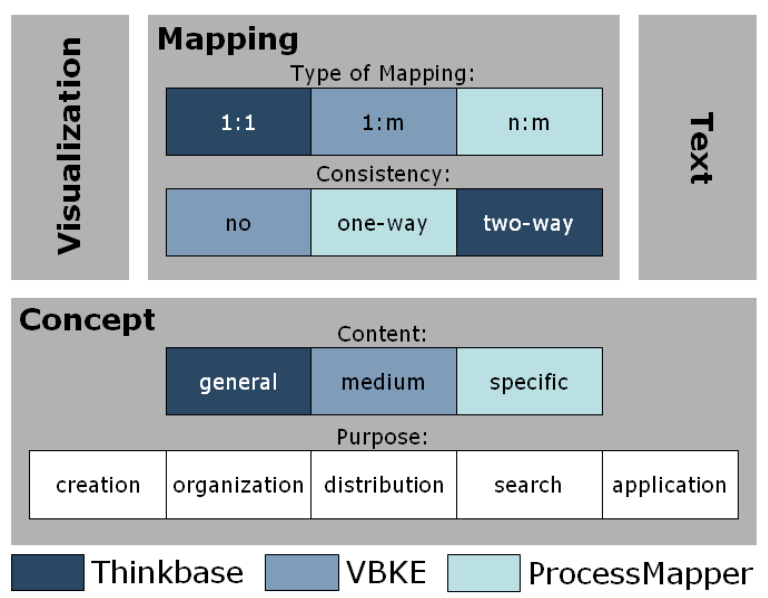

Figure 3. Design of the three prototypes

A crucial component is the mapping between the two knowledge representations. This component determines how the two representations are linked together and how they influence each other. The two most important parameters of this component are the type of mapping and the consistency between both representations. The type of mapping is the core of the mapping component and describes how the visual entities of the visualization are mapped to the textual entities of the wiki. This can basically be done in three ways: $1: 1,1: \mathrm{m}, \mathrm{n}: \mathrm{m}$. The consistency of the mapping describes how changes in one representation affect the other representation. There are three possibilities: no consistency, one-way consistency, and two-way consistency.

Figure 3 shows a more detailed view of the Visual Wiki model. It includes four of the most important parameters (type of mapping, consistency, content, and purpose) and their possible values.

\section{Methodology}

Our model shows that there are many different possibilities to design such a Visual Wiki. Each possibility might differ in some or all of the described parameters of the four components. Furthermore some of the parameters seem to depend on each other. In order to study the proposed metaphor of the Visual Wiki the following methodology has been used.

- Literature review and model development: A literature review has been carried out which ultimately led to the Visual Wiki model as described in the previous section.

- Identification of a dependent parameter: Based on this model, which describes the different parameters of the Visual Wiki, we have chosen one parameter as a dependent parameter. The selected parameter is the purpose parameter. It is of special interest as it defines which knowledge management tasks the Visual Wiki supports (creation, organization, distribution, search, or application of knowledge). The dependent parameter is shown at the bottom in Figure 3. Our prototypes and evaluations of these prototypes will help us gauge how well each Visual Wiki scenario meets these purpose parameter values.

- Identification of independent parameters: As a next step, three of the remaining parameters have been selected according to their suggested potential to influence the dependent parameter. The selected independent parameters are: content of visualization, type of mapping, and consistency of mapping (see Figure 3).

- Prototype design and implementation: Three Visual Wikis have been designed and prototypes implemented. The three prototypes have been 
designed so that they differ in the three selected independent parameters. The coloring/shading in Figure 3 show these chosen parameters for the three prototypes. Detailed descriptions of the prototypes follow in the next section.

- Evaluation: A formal evaluation has been carried out to determine what influence the prototypes and therefore the combinations of independent parameters have on the dependent parameter.

As a result this research provides three different outcomes. First of all our model provides a classification framework, which can be used to analyze, discuss, and compare different approaches to the Visual Wiki. Secondly, our research provides a proof of concept for the implemented prototypes. Lastly, the evaluation of these prototypes provides suggestions on how the chosen combinations of the independent parameters influence the different purposes of a Visual Wiki.

\section{The Prototype Visual Wikis}

This section describes the requirements, design and prototypical implementation of the three Visual Wikis as mentioned in the previous section. A common design choice for all prototypes was the use of Thinkmap (www.thinkmap.com) for the visual representation implementation platform as it can be easily extended and integrated.

Thinkmap is a software platform for developing customized visualization interfaces. It consists of loosely coupled components which provide users the ability to retrieve a result set from data sources, and then visualize, navigate, and organize it. The Thinkmap Software Development Kit (SDK) provides ways to easily extend and adjust the suite as well as to integrate it with other web and database technologies [9].

\subsection{Thinkbase}

The first prototype, Thinkbase, is an integration of Thinkmap and Freebase (www.freebase.com). Freebase is a web application described as an open, shared database of the world's knowledge [5]. It can also be described as a semantic wiki. The three main requirements for the Thinkbase prototype according to the model in Figure 3 are: the content represents general knowledge, the type of mapping is a $1: 1$ mapping, and the consistency is in both ways.

Freebase has been chosen for this prototype as it represents very general knowledge. Furthermore it provides both read and write access to the content through an API. The Thinkbase application accesses the Freebase content using that API and represents it in a Thinkmap using a custom data source layer. As a result the visualization represents very general knowledge. In this specific case the visualization represent Freebase topics (articles) and their semantic relationships.

Figure 4 show several screenshots of Thinkbase: a) shows the general user interface (UI) with the Thinkmap on the left side and Freebase on the right. The interactive graph can be used to navigate and explore the knowledge space. The names of the relationships between nodes in the graph are shown on mouse over and some relationships are combined

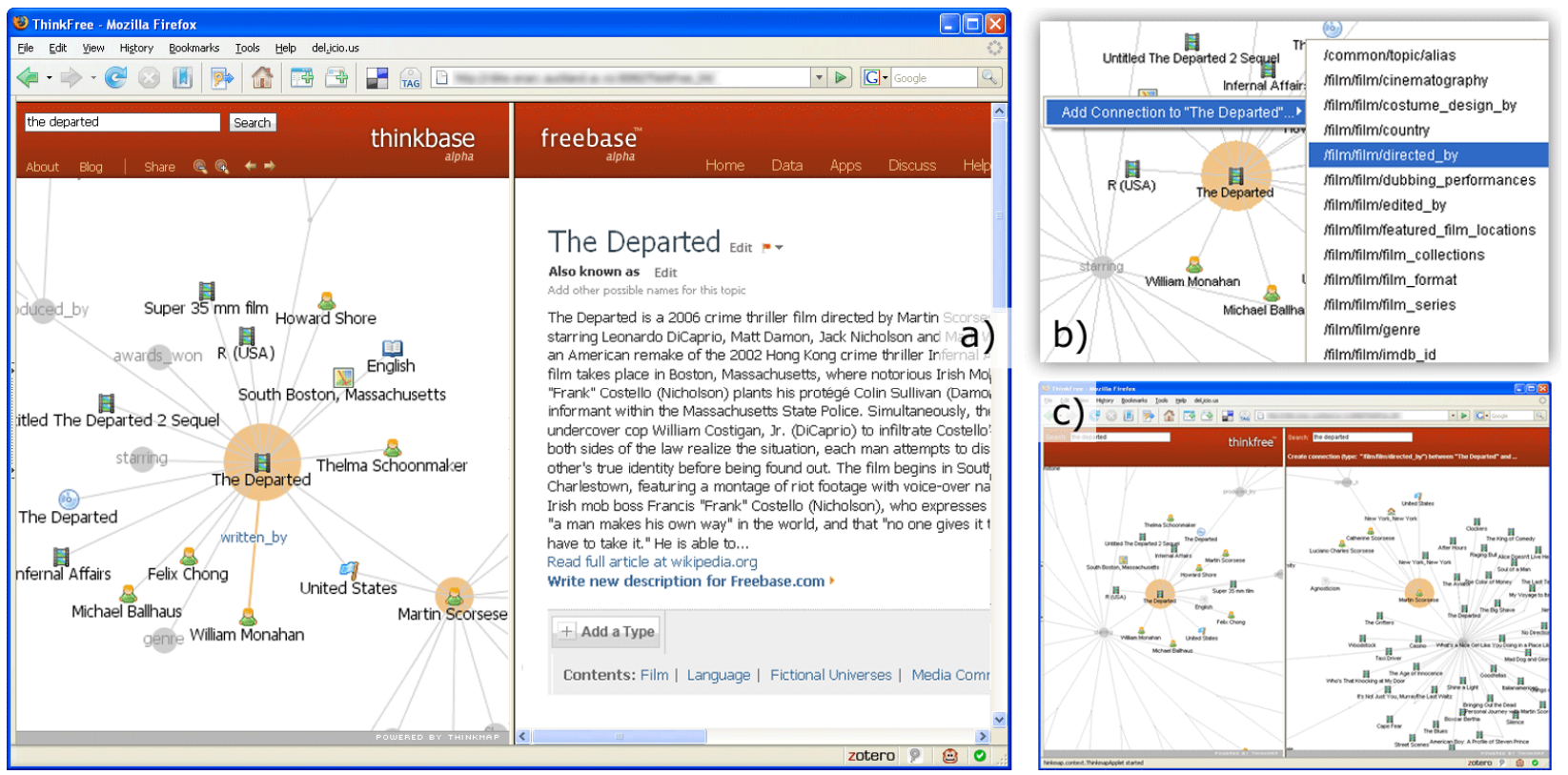

Figure 4. Thinkbase screenshots 

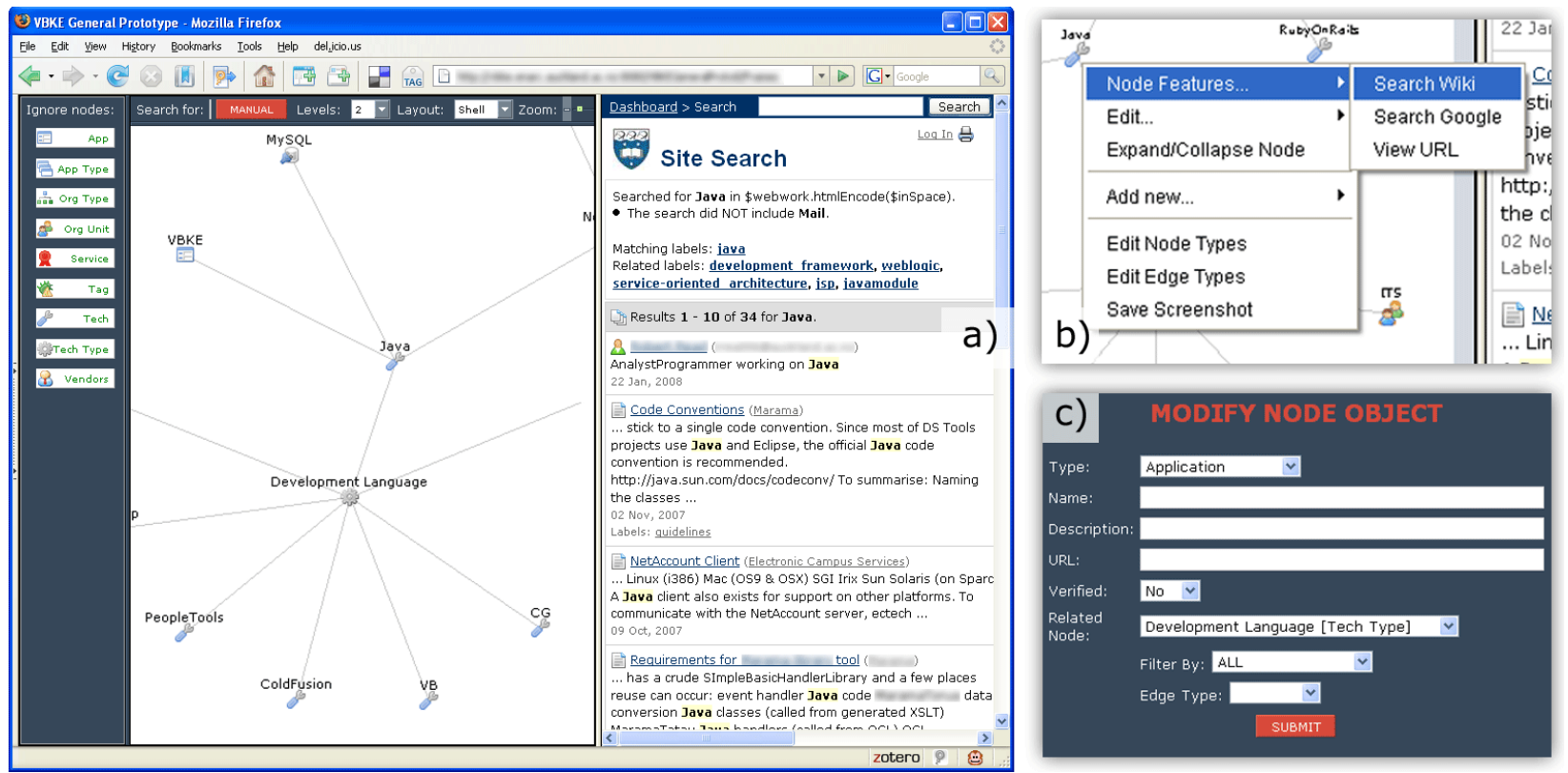

Figure 5. VBKE screenshots

using aggregation nodes. Furthermore, a context menu provides several functionalities such as the ability to further extend or collapse parts of the graph. Figure $4 \mathrm{~b}$ ) demonstrates what the context menu for adding new semantic relationships looks like, and c) shows how a second visual representation is used to establish these new relationships.

Central to the Visual Wiki is the mapping between the two representations. Thinkbase provides a 1:1 mapping. This means that each node in the visualization is mapped to one page in the textual component (Freebase) and one edge is mapped to one semantic relationship between those components. Each Freebase topic is represented in the graph using different icons depending on the type (e.g. person, movie, etc.).

As both representations receive their data from a common source (the underlying Freebase database), a two-way consistency is realized. This means that changes to both representations affect the other one. Changes to the textual representation can be achieved by editing the Freebase UI. This includes for example creating, editing, or deleting of topics and the relationships between them. The visual representation is editable as well. However, this is limited to the creation of new relationships which is done using the context menu.

\subsection{VBKE}

The second prototype, the Visual Body of Knowledge Explorer (VKBE), is an integration of Thinkmap and Confluence, a corporate wiki (www.atlassian.com). VBKE is a web application that allows visual exploration and creation of a knowledge repository combined with the ability to map to wiki pages. It was developed for internal use within the authors' institution. The three main requirements for the VBKE prototype according to the Visual Wiki model in Figure 3 are: the content represents more specific knowledge; the type of mapping is a 1:m mapping; and there is no consistency between the representations.

Figure 5 shows several screenshots of VBKE: a) shows the general UI with Thinkmap on the left side and a search result within the Confluence wiki on the right side. The visual component includes further functions such as filtering and layout functions; b) demonstrates how the context menu can be used to perform different types of searches as well as to access editing features; c) shows one of the editing forms for the visual component. In this case the modification of a node.

The visual component of VBKE has its own customized database, which describes the IT infrastructure of the institution in a graph like structure. The textual component (the Confluence wiki) is a general knowledge repository operated by the institution's IT Services. As a result the content of the visualization represents more specific knowledge as it does not represent the complete content of the textual component (like Thinkbase). However, it is still quite flexible and extendable.

The mapping between the visual and the textual component represents a 1:m mapping. This means that one node in the graph is mapped to a set of wiki pages. This set is determined by a search across the wiki space for appropriate content in the pages. 
Further mapping possibilities include a Google search result and assignment of a concrete URL.

As the visual component has its own data source, which is completely decoupled from the wiki, there is no automated consistency between the two representations. Both are editable independently. The visual component provides a role based moderated editing mechanism. That is, users can suggest changes to nodes and edges which need to be approved by a moderator. These changes do not automatically affect the wiki. In the same way changes to the wiki do not affect the visual component. However, changes to both representations might influence the mapping as search results may change.

\subsection{ProcessMapper}

The third prototype, ProcessMapper, is an integration of Thinkmap and a MediaWiki. ProcessMapper is an application which visualizes an interactive business process representation and maps it to a wiki which is intended to be a process documentation repository. The three main requirements for the ProcessMapper prototype according to the Visual Wiki model in Figure 3 are: the content represents specific knowledge; the type of mapping is a n:m mapping; and there is a one-way consistency between the representations.

Figure 6 shows several screenshots of the ProcessMapper: a) shows the general UI with an example process on the left side (in this case a university enrolment process) and a search result within the wiki on the right side; b) shows the entity specific context menu of an activity. Different searches which translate into different mappings to the wiki are possible. Figure $6 \mathrm{c}$ ) shows an alternative display of the same process. When the user navigates to a business unit, the visualization dynamically changes towards an org chart like display.

The visual language used for the process is very similar to BPMN. It consists of activities, business units, resources, and gateways. Each entity has different attributes and constraints. Business units for example have an "URL" attribute (amongst others); resources can only be connected to activities; etc. As a result the underlying metamodel is rather complex compared to the previous two prototypes and the content can represent very specific knowledge.

The mapping between the visual and the textual component represents a $\mathrm{n}: \mathrm{m}$ mapping. This is achieved in two ways: First, connected entities in the visualization can be used to perform a combined search in the wiki. Second, connected entities can be used to perform separate searches across the wiki (or open concrete URL's) in two different frames. An example of this is shown in Figure 6 a).

The ProcessMapper provides an XML interface which can be used to load business processes defined in the XML format. The required format can easily be transformed to and from BPEL (even though this is not implemented for this prototype) and therefore the tool can potentially be integrated with other BPM tools. When the process is loaded for the first time, ProcessMapper automatically creates wiki pages for the different entities based on templates. In case the XML file gets modified (e.g. new business units added) new pages will also be added to the wiki. This

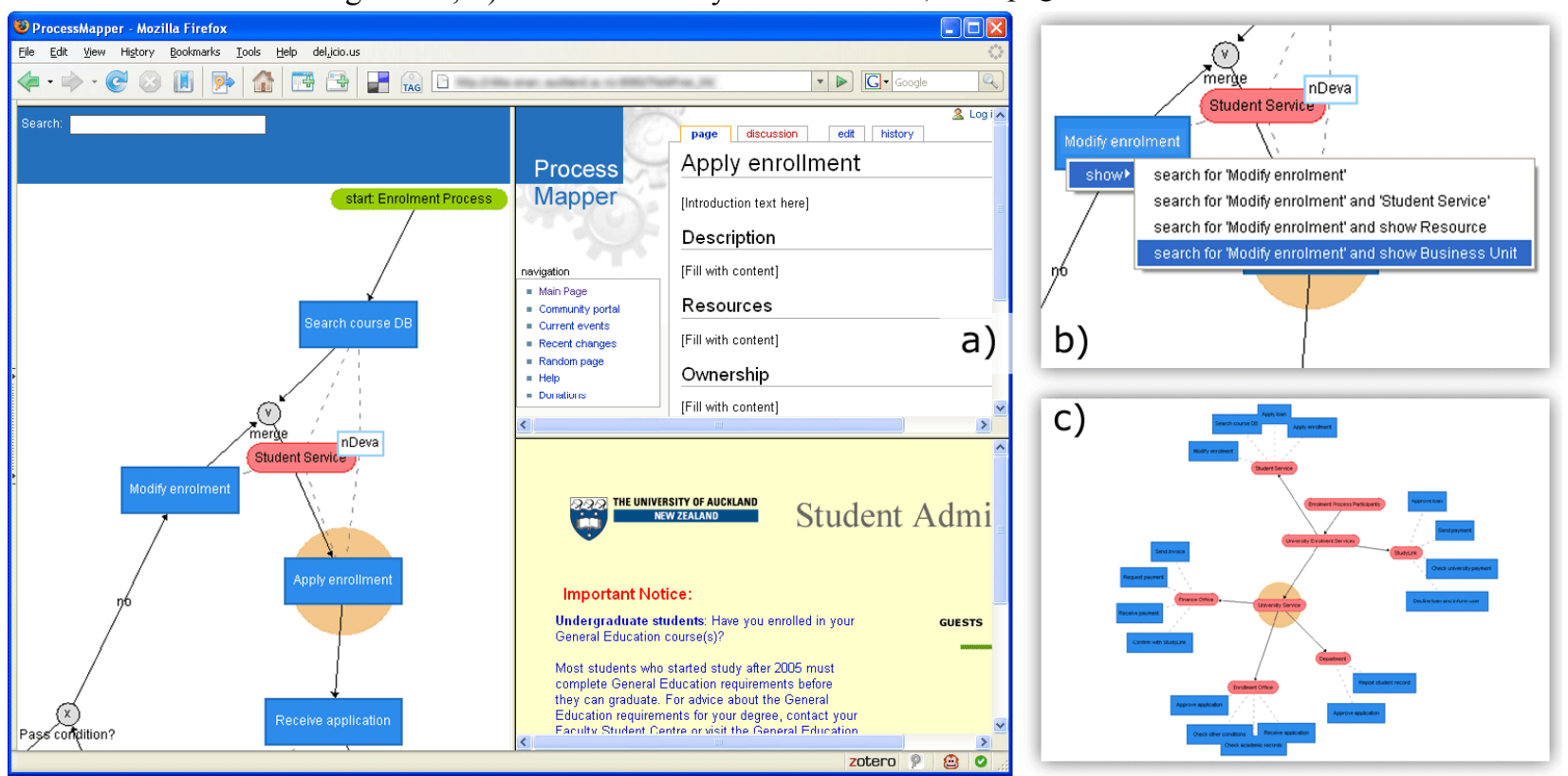

Figure 6. ProcessMapper screenshots 
results in the desired one-way consistency. Changes in the visualization will have an impact on the wiki, but not the other way. To prevent unwanted deletes, wiki pages are not deleted in case an entity is deleted in the process definition.

\section{Evaluation}

We undertook a qualitative evaluation to answer the question: how do the different Visual Wiki prototypes support and improve different knowledge management tasks (e.g. transfer of knowledge, search and navigation within a body of knowledge, etc.)? In other words: what kind of effects do the chosen independent parameters have on the dependent parameter (see Figure 3)?

During an earlier stage of the project, an informal survey was carried out amongst a small group of 5 people who were stakeholders for the development of one or all three of the prototypes. This helped us to determine suitable questions for the actual evaluation.

We then recruited a group of 14 participants for the evaluation of the three prototypes. Among the participants were 3 undergraduate students, 7 postgraduate students, and 4 staff members of the authors' institution. All three prototypes were shown to the participants together with a tutorial in order to gain familiarity with the tools. The participants then answered a set of questions for each of the prototypes as well as some general questions regarding their previous experience with wikis. The answers were gathered using an anonymous online survey tool.

All of the participants were at least "a little bit" experienced with the use of wikis, even though about $14 \%$ never used them in the corporate context (which in this case refers to the usage of wikis e.g. for project collaboration at the university). About $20 \%$ described themselves as "power users" of wikis in the public domain, $30 \%$ as "power users" in the corporate context. Power users are users which "document and collaborate on a lot of projects using wikis". Half the participants were familiar with the Confluence wiki. Freebase was less known amongst the participants. About $40 \%$ never heard of it, and only 1 participant had used it actively (write).

The questions regarding the three prototypes were structured in the same way. They were aimed to find out how much the four different tasks (creating, organizing, transfer, and search) improved as perceived by the participants. For example, participants were asked to first only consider the Confluence wiki and then rate how useful the tool is to create or organize content. Then they were asked to look at the VBKE prototype (which combines Confluence with a visualization) and rate how much the usefulness of the tool for these tasks has improved.

The first interesting result was revealed when asking the participants about the usefulness of the wikis alone. That is Freebase, Confluence, and MediaWiki. For all three tools the participants rated the usefulness of the four tasks very similarly. However, there are differences between the three tools. The usefulness of the MediaWiki for the four tasks was rated lower than the one for Confluence. Freebase was rated highest. This ranking corresponds to the degree of structure within the tools. MediaWiki is least structured, Confluence is a bit more structured (e.g. by providing spaces, labels, plugins, etc.), and Freebase with its semantics is the most structured.

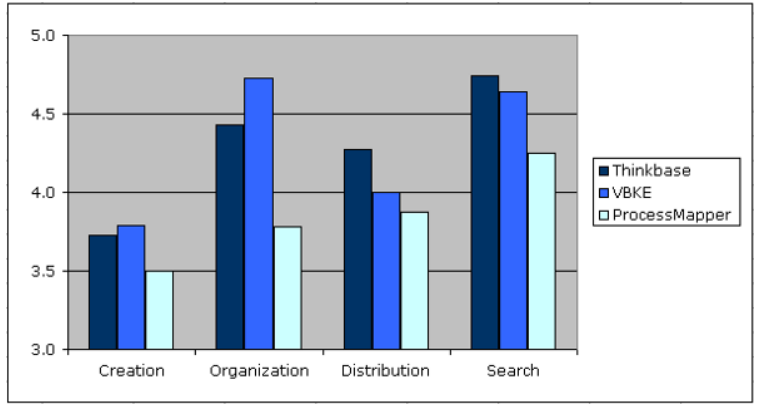

Figure 7. Results of the evaluation

The main focus of the evaluation was then on comparing these ratings with the ratings of the actual Visual Wiki prototypes. The results can be seen in Figure 7. The diagram shows the four tasks (create, organize, transfer, search) on the x-axis. The y-axis shows a scale of how much the "usefulness" of the prototypes improved on average for each of the four tasks. The value 3 corresponds to "very little" improvement, 4 to "moderate" and 5 to "high" improvement. The full scale reaches from 1 ("worse") to 6 ("very high"). Overall the results for the three prototypes are very similar. The highest improvement was perceived for the tasks of searching and organizing. The improvement in search (and navigation) relates to the benefit of visualizations as an effective method to represent knowledge rich scenarios [6]. The task of organizing is closely related to the one of searching, which could be a reason why it was rated quite high as well. The improvements in distributing knowledge were rated as somewhat less useful even though they are still significant. Lastly, the Visual Wiki concept had the least impact on the task of creating knowledge. A reason for that could be that this is a less intuitive task (as opposed for example to search) and therefore participants were not as comfortable using the visual interfaces to create new content. Interaction with creation support in the visualizations is also currently more limited than the textual wikis. Therefore, this 
particular finding might be limited to the prototypes only. Further related aspects, such the willingness of participants to collaboratively edit visualizations, were out of scope for our evaluation.

Besides the general similarities between the three prototypes as found in these results, there are also some differences between them. Most notably the ProcessMapper was rated to provide the lowest improvements for all the tasks. A reason for that could be that it is the least mature of the prototypes which means that its functionalities are limited in some ways and it also provides only empty template wiki pages and no real content. Other differences among the prototypes are: VBKE was rated highest for the task of organization for which the reason could be that it provides the most sophisticated editing functions; Thinkbase was rated highest for search and navigation for which the reason could be that the content represents very general knowledge. A specific type of search which could be called "search by exploration" was described as being "incredibly addictive" by several people who used the tool.

Further results from the survey also refer to properties of the Visual Wiki model which have not been explicitly mentioned. Participants rated the approach of Thinkbase for example most useful where the textual representation already provides some kind of semantics, while VBKE was rated more appropriate for semi-structured content. This refers to the metamodel property of the textual component.

A weakness of our evaluation is the sample size which is quite small. Furthermore, the participants are not necessarily representative for the tools' intended target user group. As at least two of the tools (Thinkbase and VBKE) are or will be used in a real setting, a more appropriate evaluation with actual users of the tools will be possible in the future.

\section{Future Work}

All three Visual Wiki prototypes have potential to be improved. Thinkbase was recently made publicly available and was received very positively by the international community. The received feedback also helps us to identify new features. This includes different visualization and layout techniques; more control over the visualization itself; and more advanced search mechanisms in order to even better navigate and understand the knowledge space. Such improvements would again put emphasize on search and navigation - an area where Thinkbase was already rated as very useful in our evaluation.

VBKE was also received very positively within the authors' institution where it is intended for internal use as an IT infrastructure exploration tool. A production project is currently being initialized in order to further develop VBKE. The ProcessMapper tool was meant as a purely proof of concept prototype which could for example further be developed to explore different mapping mechanisms.

Future work also includes further improving our Visual Wiki conceptual model (see section 3). We see this model as a starting point rather than a final or complete one. Based on the model, further new and existing prototypes of Visual Wikis could be designed, implemented and evaluated in a similar way as has been done in this research.

\section{Conclusions}

There are three outcomes of our research so far: The proposed Visual Wiki model, a proof of concept for three implemented prototypes of a Visual Wiki, and an evaluation of those prototypes.

Based around the metaphor of a Visual Wiki, a literature review has been carried out. Two research areas were of special interest to us here: new emerging internet tools and technologies and their applications in the corporate context; and the area of information and knowledge visualization and their applications to the field of knowledge management. Our literature review suggests synergic effects between those two fields which can be elaborated for knowledge management tools. However it also shows a lack of integration within existing approaches. The literature review ultimately led to the proposal of a conceptual Visual Wiki model. This model can be used to design, analyze, and discuss the different existing as well as new approaches to Visual Wikis.

Based on our model, three prototypes have been designed and implemented. Those prototypes provide a proof of concept for the Visual Wiki metaphor. Most notably it has been demonstrated that different kinds of mappings between the two representations are possible. This crucial component was missing in most of the existing approaches to a Visual Wiki.

Finally our initial evaluation shows not only that the prototypes are practicable to implement (proof of concept) but also in what kind of context they are most likely to be useful. This is possible as the proposed model can be used to choose different sets of parameters for the prototypes and then evaluate them according to those parameters. The selected parameter of interest for this research was the purpose parameter. The results of the evaluation suggest that the Visual Wiki metaphor in general improves tasks related to search and organization of information. It furthermore suggests some smaller but noticeable correlation between the chosen independent parameters and specific purposes of the 
Visual Wiki. Thinkbase appears to be most useful for search (navigation) within a knowledge space. VBKE appears to be most useful for organizing knowledge.

All three outcomes, the model, the prototypes and the evaluation, are extendable and can, as described in section 7 , be used to further explore the Visual Wiki metaphor and its applications in the field of knowledge management.

\section{References}

[1] Anderson, C., The Long Tail: Why the Future of Business Is Selling Less of More. 2006: Hyperion.

[2] Ankolekar, A., et al., The two cultures: mashing up web 2.0 and the semantic web. Proc. of the 16th international conference on World Wide Web. 2007: p. 825-834.

[3] Auer, S., S. Dietzold, and T. Riechert, OntoWiki-A Tool for Social, Semantic Collaboration. Proc. of the 5th International Semantic Web Conference (ISWC'05). 4273: in LNCS, Springer, 2006, p. 736-749.

[4] Berners-Lee, T., J. Hendler, and O. Lassila, The semantic Web. Scientific American. 2001. 284(5): p. 34-43.

[5] Bollacker, K., R. Cook, and P. Tufts, Freebase: A Shared Database of Structured General Human Knowledge. Proc. of the national conference on Artificial Intelligence, 2007. 22(2): p. 1962.

[6] Burkhard, R., Towards a Framework and a Model for Knowledge Visualization: Synergies between Information and Knowledge Visualization. LNCS 3426. Springer: Berlin, Heidelberg, New York, 2005.

[7] Chatzkel, J., Conference report 2006 KMWorld Conference Review. Journal of Knowledge Management, 2007. 11(4): p. 159-166.

[8] Davis, M. Semantic Wave 2008 Report: Industry Roadmap to Web 3.0 \& Multibillion Dollar Market Opportunities. 2008 [29-02-2008]; http://project10x.com.

[9] Design, P. The Thinkmap White Paper. [2008-03-08]; http://www.thinkmap.com/.

[10] Dotsika, F. and K. Patrick, Towards the new generation of web knowledge. VINE: The journal of information and knowledge management systems, 2006. 36(4): p. 406-422.

[11] Eppler, M. and R. Burkhard, Knowledge Visualization. in Encyclopedia of Knowledge Management, Idea Group, 2005.

[12] Jaeschke, G., M. Leissler, and M. Hemmje, Modeling Interactive, 3-Dimensional Information Visualizations Supporting Information Seeking Behaviors. in Knowledge and Information Visualization: Searching for Synergies. Springer 2005: p. 119-135.

[13] Keim, D.A., Information visualization and visual data mining. IEEE Transactions on Visualization and Computer Graphics, 2002. 8(1): p. 1-8.

[14] Keller, T. and S.O. Tergan, Visualizing Knowledge and Information: An Introduction. in Knowledge and Information Visualization: Searching for Synergies. Springer 2005: p. 1-23.

[15] Lengler, R. and M.J. Eppler, Towards a Periodic Table of Visualization Methods of Management. Proc. of the Conference on Graphics and Visualization in Engineering, 2007, pp. 1-6.

[16] McAfee, A.P., Enterprise 2.0: the dawn of emergent collaboration. MIT Sloan Management Review, 2006. 34(3): p. 38-38.

[17] McKinsey, How businesses are using Web 2.0: A McKinsey Global Survey. The McKinsey Quarterly 2007.

[18] Mika, P., Flink: Semantic Web technology for the extraction and analysis of social networks. Journal of Web Semantics, 2005. 3(2-3): p. 211-223.

[19] O'Reilly, T., What Is Web 2.0: Design Patterns and Business Models for the Next Generation of Software. O'Reilly Media 2005.

[20] Shneiderman, B., The eyes have it: a task by data type taxonomy for information visualizations. Proc. IEEE Symp. Visual Languages, IEEE CS Press, 1996: p. 336-343.

[21] Tapscott, D., Winning with the Enterprise 2.0. New Paradigm Learning Corporation, 2006.

[22] Tapscott, D. and A.D. Williams, Wikinomics: how mass collaboration changes everything. 2006: Portfolio.

[23] Tergan, S.O. and T. Keller, Knowledge and Information Visualization: Searching for Synergies. LNCS 3426. Springer, 2005.

[24] Tredinnick, L., Web 2.0 and Business: A pointer to the intranets of the future? Business Information Review, 2006. 23(4): p. 228.

[25] Ullman, A.J. and J. Kay, WikiNavMap: a visualisation to supplement team-based wikis. Conference on Human Factors in Computing Systems, 2007. p. 2711-2716.

[26] Vail, E.F., Knowledge Mapping: Getting Started with Knowledge Management. Information Systems Management, 1999. 16(4): p. 1-8.

[27] Viégas, F.B., et al., Many Eyes: A Site for Visualization at Internet Scale. Proc. of Infovis, 2007. 